Article

\title{
Lateral Momentum Fluxes at the Confluence of the Negro and Solimões Rivers
}

\author{
Adriano Coutinho de Lima \\ Division of Field Engineering for the Environment, Hokkaido University, Sapporo 0608628, Japan; \\ adriano@eng.hokudai.ac.jp; Tel.: +81-011-706-6187
}

Received: 13 November 2018; Accepted: 19 December 2018; Published: 23 December 2018

check for updates

\begin{abstract}
Hydrodynamic zones of river confluences are remarkable not only for the turbulent mixing induced by the shear layer at the center of the mixing interface but also for the lateral momentum fluxes associated with channel topography. Detailed characterizations of lateral momentum transfers in river confluences, however, are few. In this study, contributions to the lateral momentum fluxes in the confluence of the Negro and Solimões rivers in Brazil were calculated based on a comprehensive set of field data. Results show that the lateral fluxes by the mean flow exceed the turbulent fluxes by two orders of magnitude. Furthermore, the Reynolds stress along the far field of the Solimões side of the Amazon channel scales with or surpasses the Reynolds stress at the interface with the Negro side. The importance of the shear layer in the lateral mixing is thus overshadowed by the competing hydrodynamic processes. This configuration partially explains the long distance required to complete the mixing of the waters of the two tributary rivers.
\end{abstract}

Keywords: river confluence; turbulence; momentum flux; field data

\section{Introduction}

The confluence of the Negro and Solimões rivers which feeds into the Amazon River in Brazil (Figure 1 [1]) is remarkable for the different geomorphological and dynamic characteristics of the two tributary rivers, the long time (more than $30 \mathrm{~h}$ ) and long distance (more than $100 \mathrm{~km}$ ) required to complete mixing [2], and the large magnitude of the discharges involved: the long-term combined discharge of the Negro and Solimões rivers corresponds to approximately 10\% of the Earth's freshwater discharge in the oceans. Along the hydrological year, the velocity of the Solimões river near the confluence varies from approximately 2 to 14 times that of the Negro river [3]. The velocity inflection in the center of the shear layer downstream from where the rivers merge enhances the transverse mixing and the Kelvin-Helmholtz instability. In spite of the sharp velocity gradient, the lateral expansion of the shear layer and the mixing interface downstream of the junction is limited by hydrodynamic mechanisms associated with geometric characteristics of the channel, as commonly observed in river confluences. The shear layer is typically defined as a zone of intense turbulence that is generated by a velocity inflection and is thus associated with hydrodynamic characteristics, whereas the mixing interface is associated with properties such as turbidity and color $[4,5]$.

The stability characteristics of lateral shear layers in shallow flows at channel junctions, composite channels, channels with laterally varied bottom roughness, or channels with lateral vegetation or pile dikes have been studied extensively by means of linear stability analysis [6-14]. In the frameworks of References [6-14], the channel planform is parallel, and a linear stability analysis can successfully explain 
the effects of inertial and gravitational forces, bed shear stress, and shear layer turbulent stress on the stability of the lateral shear flows. In parallel channels with shallow mixing interfaces, turbulence is characterized by two distinct scales: sub-depth turbulence, which is associated with the bed shear stress, and the large-scale turbulence associated with large horizontal eddies that develop in the shear layer [15]. The lateral expansion of the shear layer is limited because solid boundaries in the channel act as stabilizing mechanisms that lock the amplitude of the large-scale eddies into a finite-equilibrium state [15].

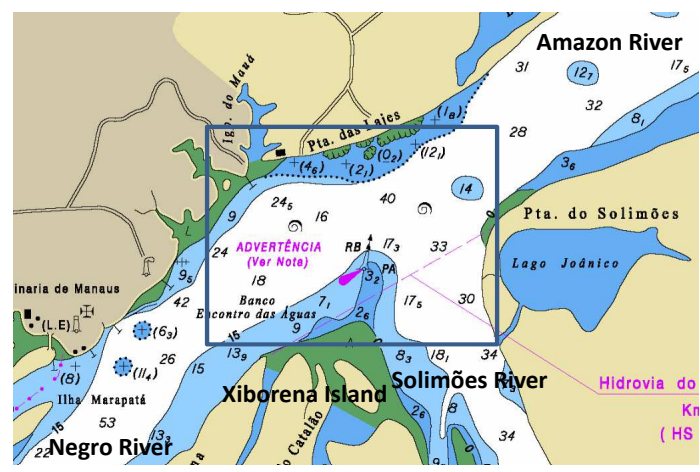

(a)

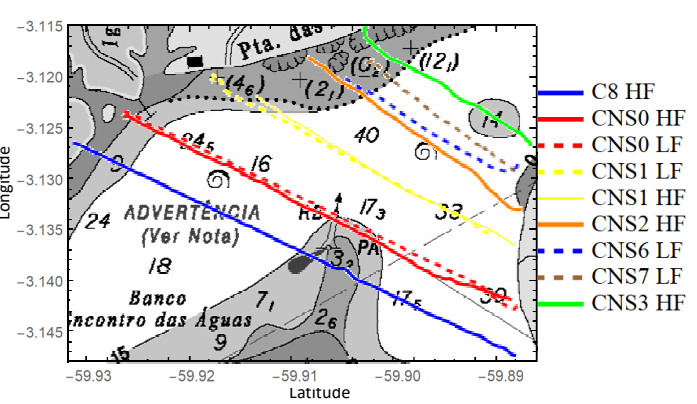

(b)

Figure 1. Nautical chart (source: Brazilian Navy [1]) showing the confluence of the Negro and Solimões rivers. The area within the blue square in (a) is enlarged in (b), where the locations of the transects of a collection of data by the CLIM-Amazon Project are shown. The numbers indicate the depth in meters of the level above which the rivers remain for approximately $90 \%$ of the time.

At river confluences, particularly in mega rivers such as the Negro and Solimões, although the shear layer is unstable, the effect of this instability is weakened by the dissipation of energy resulting from turbulence generated upstream of the junction. Furthermore, the typical oblique angle between the two tributary rivers and the complex bed topography and channel planform not only induces further turbulence but also generates considerable lateral fluxes of momentum. These fluxes of momentum inhibit the lateral expansion of the large eddies arising from the instability of the shear layer, and they must be taken into account for an accurate description of the hydrodynamic aspects of confluence mixing interfaces [5]. Until recently, however, little data were available on the components of lateral momentum fluxes in mixing interfaces [5]. In this study, these components were determined for the Negro and Solimões confluence hydrodynamic zone (CHZ) using secondary data from field campaigns of the CLIM-Amazon project conducted on 31 October 2014 and 29-30 April 2015 [16,17].

\section{Methodology}

The colder and denser waters of the Solimões river partially flow underneath the waters of the Negro river, resulting in a complex three-dimensional mixing interface. However, in the present research, a two-dimensional model of fluid motion was used, which is reasonable for a scaling analysis. 
The momentum equations in the streamwise and lateral directions ( $x$ and $y$, respectively) and the continuity equation are [18]

$$
\begin{aligned}
\frac{\partial(h \overline{\langle u\rangle})}{\partial t}+\frac{\partial\left(h \overline{\langle u\rangle}^{2}\right)}{\partial x}+\frac{\partial(h \overline{\langle u\rangle} \overline{\langle v\rangle})}{\partial y}=-g h \frac{\partial z_{s}}{\partial x}+\frac{1}{\rho} \tau_{b x}+\frac{\partial\left(h \tau_{x x}\right)}{\partial x}+\frac{\partial\left(h \tau_{x y}\right)}{\partial y} \\
\frac{\partial(h \overline{\langle v\rangle})}{\partial t}+\frac{\partial(h \overline{\langle u\rangle} \overline{\langle v\rangle})}{\partial x}+\frac{\partial(h \overline{\langle v\rangle})}{\partial y}=-g h \frac{\partial z_{s}}{\partial y}+\frac{1}{\rho} \tau_{b y}+\frac{\partial\left(h \tau_{x y}\right)}{\partial x}+\frac{\partial\left(h \tau_{y y}\right)}{\partial y} \\
\frac{\partial z_{s}}{\partial t}+\frac{\partial(h \overline{\langle u\rangle})}{\partial x}+\frac{\partial(h \overline{\langle v\rangle})}{\partial y}=0
\end{aligned}
$$

where $u$ and $v$ are the velocity components in the streamwise $(x)$ and lateral $(y)$ directions, respectively, $t$ is time, $h$ is the flow depth, $z_{S}$ is the free surface elevation, $g$ is the gravitational acceleration, $\rho$ is the density of water, $\tau_{b i}(i=x, y)$ is the bed shear stress, and $T_{i j}$ is a stress component which includes viscous friction, turbulent friction, and differential advection. The overline denotes depth-averaged variables and the angled brackets denote a slowly varying mean.

The bed shear stress is expressed in the form

$$
\tau_{b i}=\rho C_{f} \overline{\left(u^{2}+v^{2}\right)^{1 / 2}\left|\left\langle u_{i}\right\rangle\right|}
$$

where $C_{f}$ is the bed friction coefficient, and $\left(u_{x}, u_{y}\right)=(u, v)$. From Gualtieri et al. [16], the values of $\left|\vec{\tau}_{b}\right|$, $\vec{\tau}_{b}=\left(\tau_{b x}, \tau_{b y}\right)$, reached up to 7 and 10 Pa under relatively low (31 October 2014) and high (29-30 April 2015) flow conditions on the Solimões side of the Amazon channel at the scour hole. In contrast, the lowest values of bed shear stress (approximately zero) were located on the Negro side of the Amazon channel and in the zones of flow stagnation and flow separation on the Solimões side.

Neglecting the viscous friction, which is appropriate for highly turbulent flows, $T_{i j}$ is expressed in the form

$$
T_{i j}=-\overline{\left\langle u_{i}^{\prime} u_{j}^{\prime}\right\rangle}+\overline{\left(u_{i}-\overline{u_{i}}\right)\left(u_{j}-\overline{u_{j}}\right)},
$$

where the primes indicate variations about the slowly varying mean.

In parallel channels with shallow mixing interfaces, the lateral momentum transfer is driven by turbulence, and the system of Equations (1)-(5) can take simpler forms which reasonably describe the mean or basic flow. At oblique river confluences, in contrast, the magnitudes of the momentum flux by the average flow $\overline{\langle u\rangle} \overline{\langle v\rangle}$ and by the differential advection $\overline{\left(u_{i}-\overline{u_{i}}\right)\left(u_{j}-\overline{u_{j}}\right)}$ are large and compete with the magnitudes of the momentum flux by turbulence $\overline{\left\langle u_{i}^{\prime} u_{j}^{\prime}\right\rangle}$. In this work, the components of these three momentum fluxes under $y$-derivatives in Equation (1) were quantified for transects located up to $4 \mathrm{~km}$ downstream of the merge, corresponding to the confluence hydrodynamic zone (CHZ). To the author's knowledge, this quantification using recent data from the Negro-Solimões confluence is novel. Within the $\mathrm{CHZ}$, hydrodynamic and morphodynamic processes are affected by (1) the planform and junction angle of confluence, (2) the momentum ratio of the merging tributaries, and (3) the concordance between the elevation of the bed of the tributaries at the entrance of the confluence [19-21]. The transects shown in Figure 1 correspond to cross-sectional sets of locations where flow velocities and flow depth (among others) were measured as part of the CLIM-Amazon Project. There are a total of nine transects, four of which correspond to relatively low flow conditions (data collected in October 2014, referred to as LF) and five transects corresponding to relatively high flow conditions (data collected in April 2015, referred to as HF). Some transects correspond to nearly coincident locations under low and high flow conditions, with slight shifts associated with positioning at high currents. The median distance between adjacent measuring 
points in a transect is approximately $1.40 \mathrm{~m}$, and the distance between transects is on the order of $10^{2} \mathrm{~m}$. Detailed descriptions of the CLIM-Amazon Project's field study on the Negro-Solimões confluence are found in References [16,17,21].

Figure 2 depicts the velocities $\bar{u}$ and $\overline{\langle u\rangle}$ for the transects CNS1 HF and CNS7 LF. Figure 2 illustrates the procedures that were adopted in this study. The quantities in angled brackets in Figure 2 and thereafter were calculated by averaging over a $100 \mathrm{~m}$ length, thus formally distinct from a slowly varying average but yielding similar quantitative results. A distance of $100 \mathrm{~m}$ was chosen to include few turbulent structures. The regularity of fluctuations along the distance indicates structured turbulence patterns. The origin of the distance on the horizontal axes of the plots in this paper corresponds to the data collection point in each transect located closest to the Negro side of the Amazon channel.

Figure 3 depicts the bed profiles of transects C8 HF, CNS7 LF, and CNS3 HF. The talwegs of each tributary remain noticeable in the downstream end of the CHZ (Figure 3), separated by a submerged prolongation of the downstream tip of Xiborena island, a bar island which splits the Negro and the Solimões rivers just upstream of the confluence. This peak between the two talwegs partially obstructs the mixing of waters from the two tributaries.

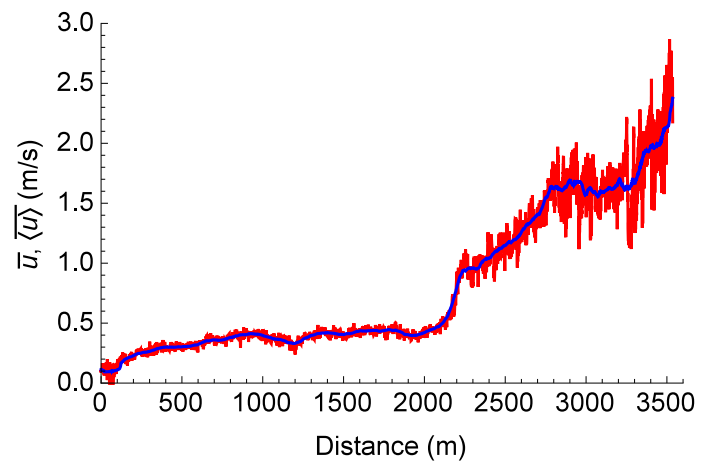

(a) CNS1 HF

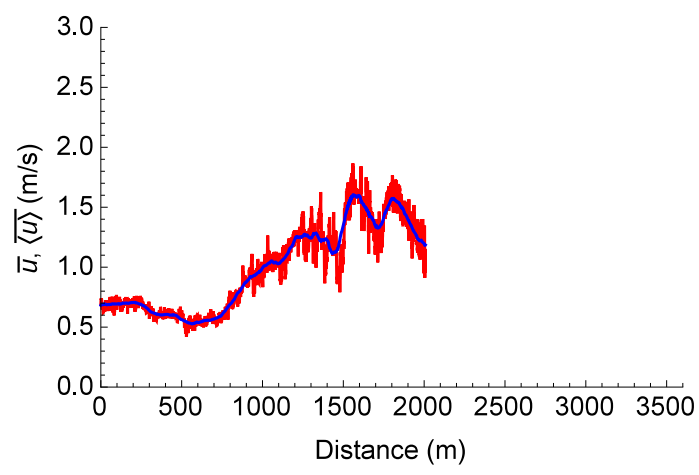

(b) CNS7 LF

Figure 2. Lateral profiles of depth-averaged velocities $\bar{u}$ (red) and $\overline{\langle u\rangle}$ (blue).

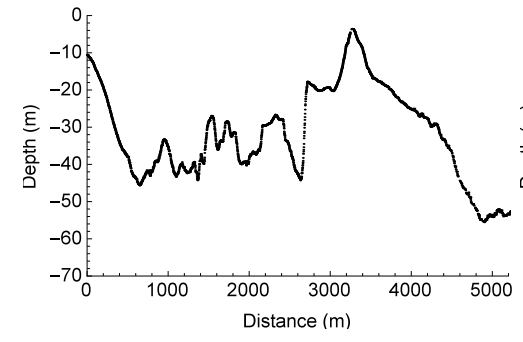

(a) $\mathrm{C} 8 \mathrm{HF}$

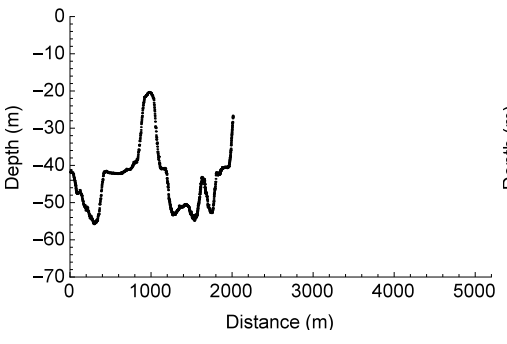

(b) CNS7 LF

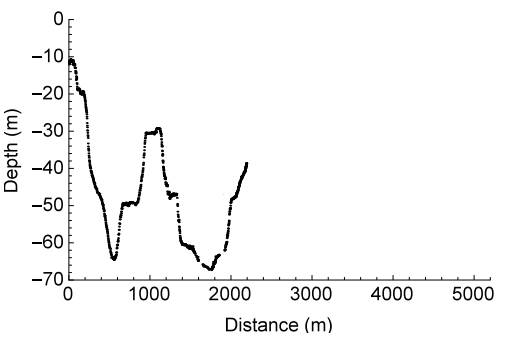

(c) CNS3 HF

Figure 3. Lateral profiles of flow depth.

\section{Results and Discussion}

Figure 4 depicts the depth- and length-averaged velocity components $\overline{\langle u\rangle}$ and $\overline{\langle v\rangle}$ along the sampling transects shown in Figure 1. The point where $\overline{\langle v\rangle}$ is zero is around the center of the mixing interface. The plots show that the magnitudes of both the streamwise and the lateral components of the velocity on the Solimões side are significantly larger than those on the Negro side. The Solimões side of the channel 
undergoes a sharp bending, whereas the Negro side remains approximately straight (Figure 1). This is reflected by the particularly strong lateral velocity component $(\overline{\langle v\rangle})$ on the Solimões side.

The lateral gradient of the velocity magnitude extends to the downstream end of the $\mathrm{CHZ}$ (Figure $4 \mathrm{~d}, \mathrm{i})$. However, the sharp lateral gradient of the values of $\overline{\langle u\rangle}$ along the center of the mixing interface diffuses along the downstream direction (sequences in Figure 4a-i), and a consistent downstream increase in the magnitude of $\overline{\langle u\rangle}$ on the Negro side is observed. Figure 4i shows a particular reduction in the absolute value of $\overline{\langle v\rangle}$ on the Solimões side, which indicates realignment of the flow after overcoming the sharp bend on the Solimões side.

Given the large magnitudes of $\overline{\langle u\rangle}$ and $\overline{\langle v\rangle}$ on the Solimões side, it follows that the momentum flux by the depth- and length-averaged flow $\overline{\langle u\rangle} \overline{\langle v\rangle}$ is considerable on this side, whereas it is close to zero on the Negro side, as shown in Figure 5. The negative values of $\overline{\langle u\rangle} \overline{\langle v\rangle}$ on the Solimões side indicate inward momentum transfer. In the sequences in Figure 5a-i, the magnitudes of $\overline{\langle u\rangle} \overline{\langle v\rangle}$ increase and then decrease on the Solimões side, where the maximum reached under the relatively high flow condition is 1.5-2 times larger than that under the relatively low flow condition. The substantial decay of $\overline{\langle u\rangle} \overline{\langle v\rangle}$ in Figure $5 \mathrm{~h}-\mathrm{i}$ illustrates the rapid realignment of the Solimões side. The Amazon channel is less sinuous downstream of CNS3 HF (Figure 5i), suggesting a weaker momentum transfer combined with a smooth lateral gradient of $\overline{\langle u\rangle}$ for several kilometers downstream of the CHZ.

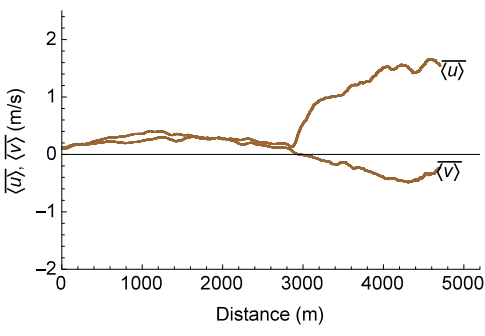

(a) CNSO LF

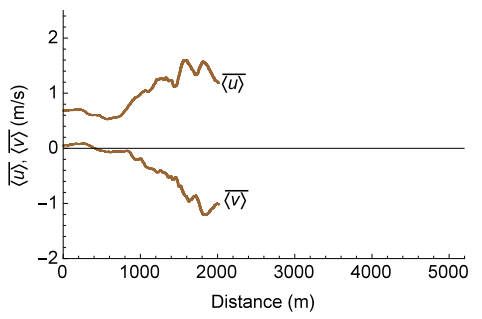

(d) CNS7 LF

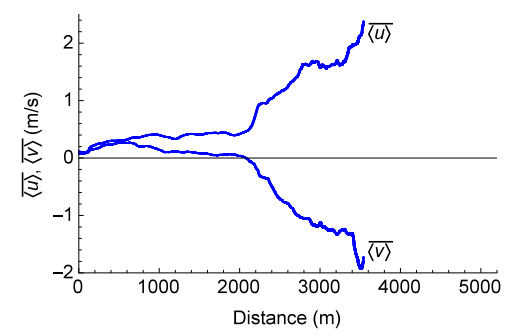

(g) CNS1 HF

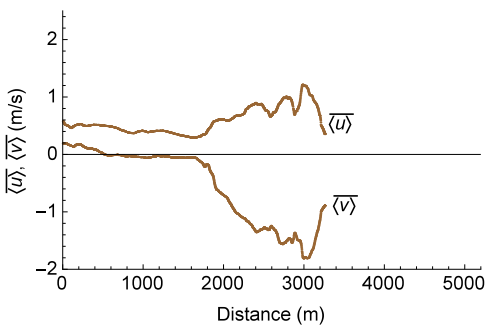

(b) CNS1 LF

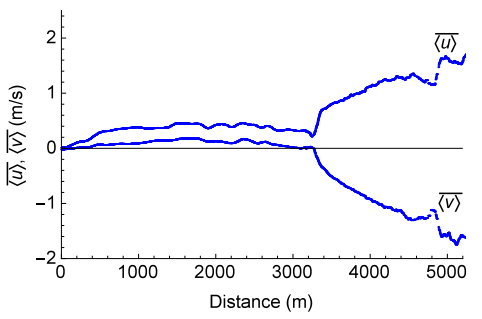

(e) $\mathrm{C} 8 \mathrm{HF}$

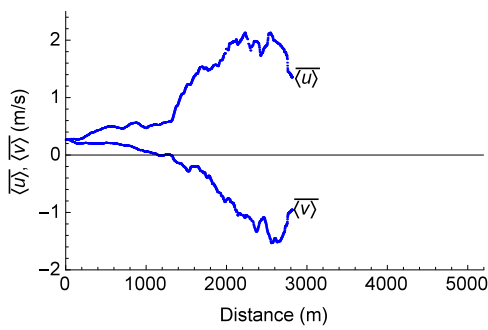

(h) CNS2 HF

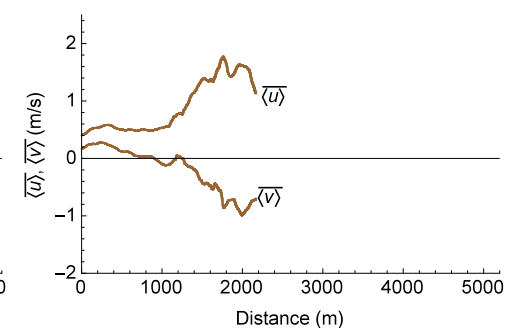

(c) CNS6 FL

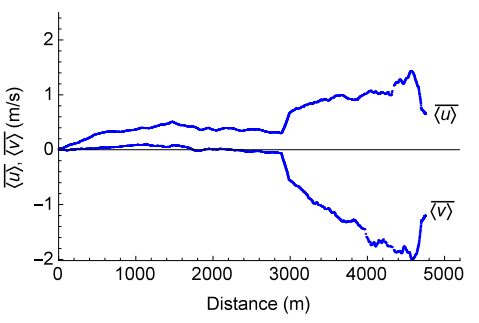

(f) CNSO HF

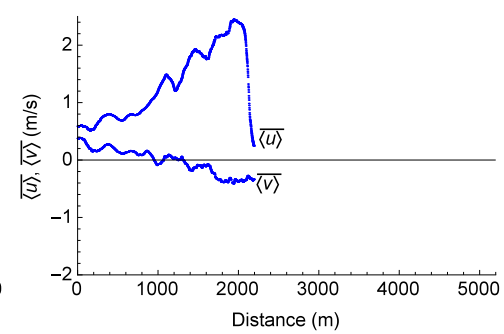

(i) CNS3 HF

Figure 4. Velocity components $\overline{\langle u\rangle}$ and $\overline{\langle v\rangle}$ across the sampling transects. The brown and blue plots correspond to the relatively low and high flow campaigns, respectively. 


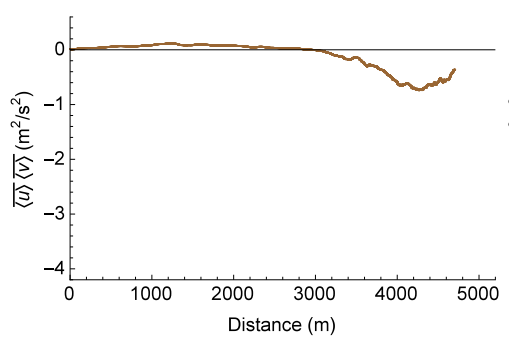

(a) CNSO LF

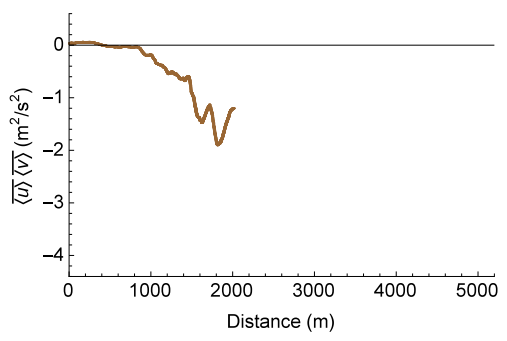

(d) CNS7 LF

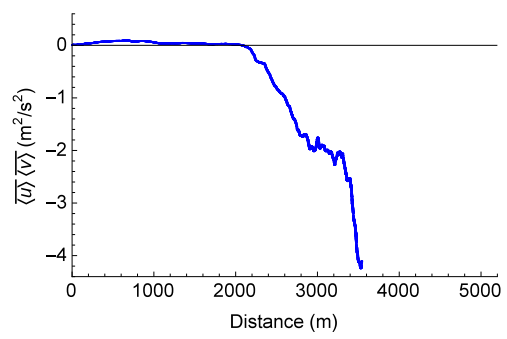

(g) CNS1 HF

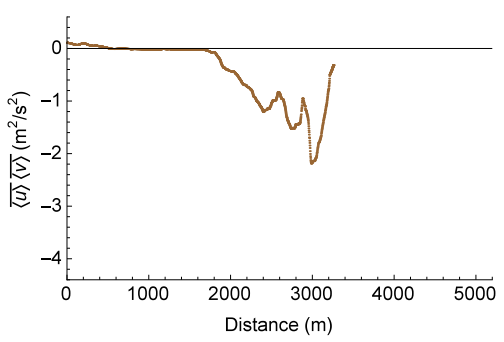

(b) CNS1 LF

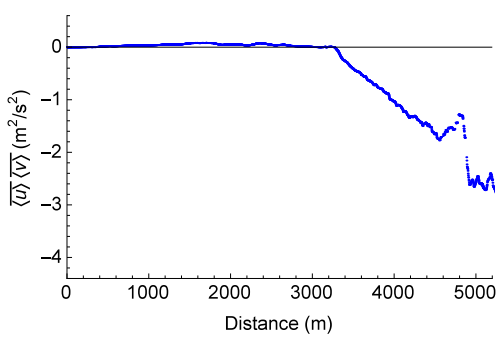

(e) $\mathrm{C} 8 \mathrm{HF}$

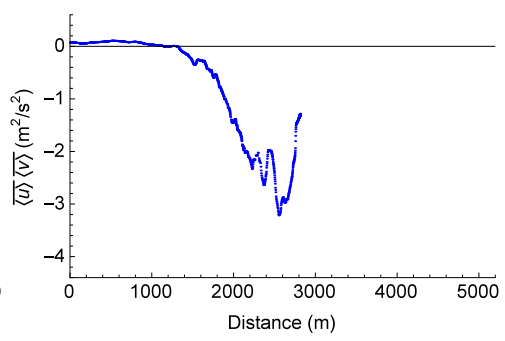

(h) CNS2 HF

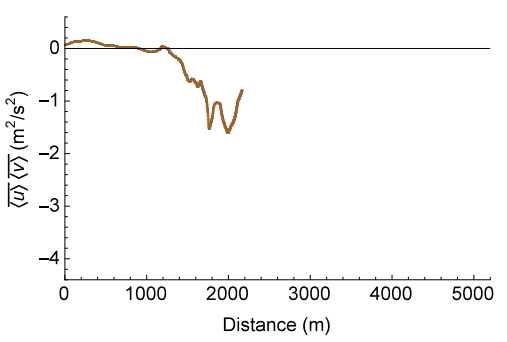

(c) CNS6 LF

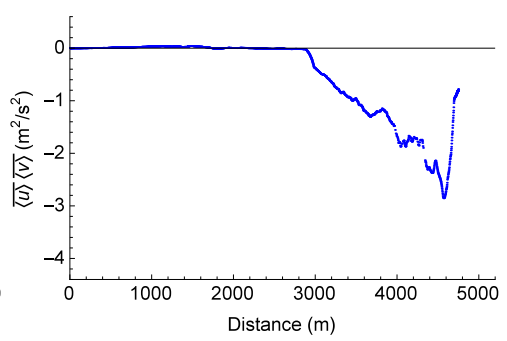

(f) CNSO HF

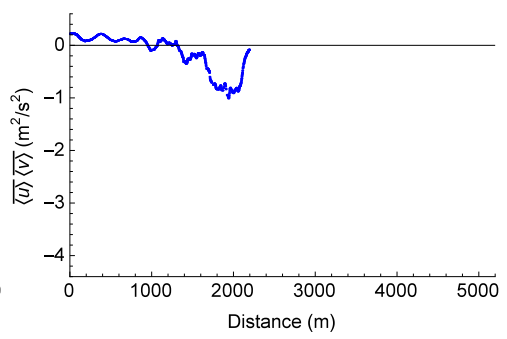

(i) CNS3 HF

Figure 5. Profiles of the momentum flux by the depth- and length-averaged flow $\overline{\langle u\rangle} \overline{\langle v\rangle}$ across the sampling transects. The brown and blue plots correspond to relatively low and high flow conditions, respectively.

The momentum flux by the depth- and length-averaged flow $\overline{\langle u\rangle} \overline{\langle v\rangle}$ is the dominant mechanism of lateral momentum transfer in the Negro-Solimões confluence, with values typically two orders of magnitude above the values of the depth-averaged momentum flux by turbulence $\overline{\left\langle u^{\prime} v^{\prime}\right\rangle}$ (Figure 6) and typically one order of magnitude above the depth-averaged momentum flux by differential advection $\overline{\left(u_{i}-\overline{u_{i}}\right)\left(u_{j}-\overline{u_{j}}\right)}$ (Figure 7). Figure 6 depicts lateral profiles of the momentum flux by turbulence $\overline{\left\langle u^{\prime} v^{\prime}\right\rangle}$ for the sampling transects. The oscillatory profiles indicate that various turbulent structures coexist along the same transect. The magnitude of $\overline{\left\langle u^{\prime} v^{\prime}\right\rangle}$ on the Negro side is negligible compared with the Solimões side, where it typically reaches values on the order of $\approx 10^{-2} \mathrm{~m}^{2} / \mathrm{s}^{2}$. Figure $6 \mathrm{a}$ is characterized by particularly small magnitudes of $\overline{\left\langle u^{\prime} v^{\prime}\right\rangle}$ which increase in the downstream direction (Figure $6 \mathrm{~b}$ ). This was not verified under relatively high flow conditions, indicating that the enhancement of the turbulence by the confluence is more significant under low flow conditions. Although oscillations characterize the lateral profiles of both $\overline{\left\langle u^{\prime} v^{\prime}\right\rangle}$ and $\overline{\langle u\rangle} \overline{\langle v\rangle}$, only the latter exhibits a net gradual increase toward the far field on the Solimões side. The amplitude of the oscillations of $\overline{\left\langle u^{\prime} v^{\prime}\right\rangle}$ on the Solimões side is typically $1-2 \times 10^{-2} \mathrm{~m}^{2} / \mathrm{s}^{2}$, indicating uniform turbulent scales. However, amplitude peaks of up to $\approx 8 \times 10^{-2} \mathrm{~m}^{2} / \mathrm{s}^{2}$ occur, and they are expected to be associated with topographic features. The values of $\overline{\left\langle u^{\prime} v^{\prime}\right\rangle}$ are not particularly large 
around the center of the mixing interface, indicating that the effect of the shear layer turbulence in the lateral mixing is limited by turbulence associated with the channel bed and planform.

The lateral profiles of the momentum flux by the differential advection $\overline{(u-\bar{u})(v-\bar{v})}$ (Figure 7) resemble those of $\overline{\langle u\rangle} \overline{\langle v\rangle}$ (Figure 5), although the values of the former are typically one order of magnitude smaller than the vales of the latter. The sum $\overline{\langle u\rangle} \overline{\langle v\rangle}+\overline{(u-\bar{u})(v-\bar{v})}$ is referred to as the lateral momentum transfer by the mean flow. The lateral momentum transfer owing to differential advection thus peaks in the far field on the Solimões side, in contrast with the limited motion in the center of the mixing interface. The values of $\overline{(u-\bar{u})(v-\bar{v})}$ were calculated assuming a logarithmic velocity distribution along the vertical direction (Equation (6)) and using the different values of bottom roughness for the Negro and Solimões sides obtained from Nordin et al. (1977) [22],

$$
u_{i}=u_{i}^{*} \frac{1}{\kappa} \ln \frac{z}{z_{0}}
$$

where $u_{i}^{*}=\left(\tau_{b i} / \rho\right)^{1 / 2}(i=x, y)$ is the friction velocity, $z$ is the vertical direction, and $z_{0}$ is the elevation, slightly above the river bed, where the logarithmic distribution of $u_{i}$ assumes the value of zero. The elevation $z_{0}$ is calculated as $10 \%$ of the bed sediment diameter $d_{8} 4$ [16,23] (i.e., the bed sediment diameter for which $84 \%$ is finer), taken as $0.0625 \mathrm{~mm}$ on the Negro side and $0.350 \mathrm{~mm}$ on the Solimões side [22].

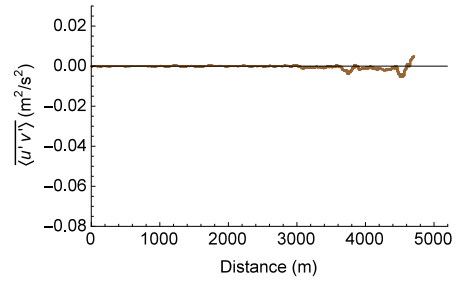

(a) CNSO LF

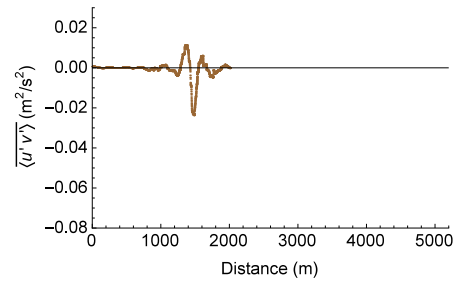

(d) CNS7 LF

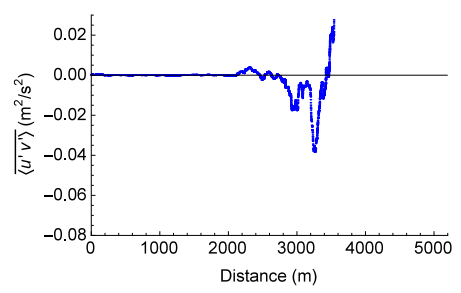

(g) CNS1 HF

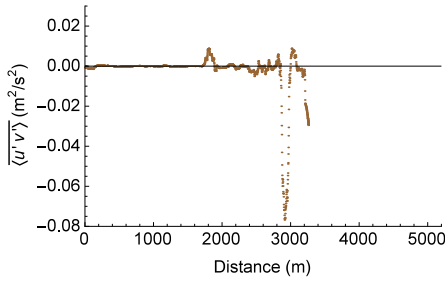

(b) CNS1 LF

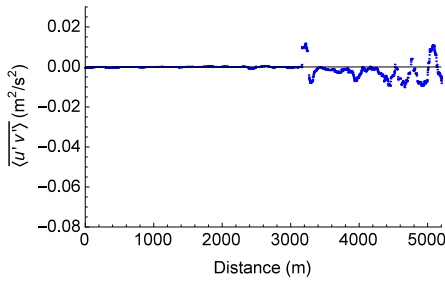

(e) $\mathrm{C} 8 \mathrm{HF}$

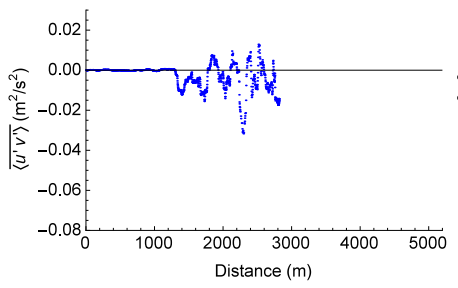

(h) CNS2 HF

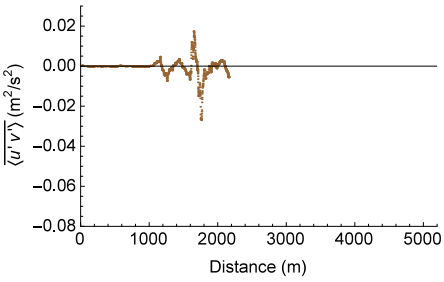

(c) CNS6 FL

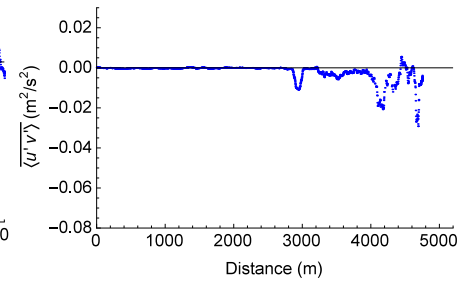

(f) CNS0 HF

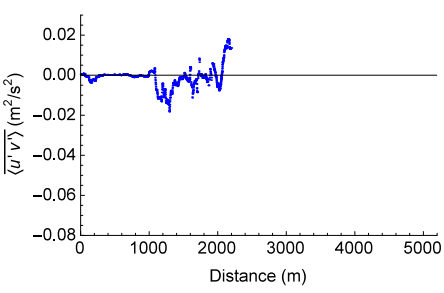

(i) CNS3 HF

Figure 6. Profiles of the momentum flux by turbulence $\overline{\left\langle u^{\prime} v^{\prime}\right\rangle}$ across the sampling transects. The brown and blue plots correspond to relatively low and high flow conditions, respectively. 


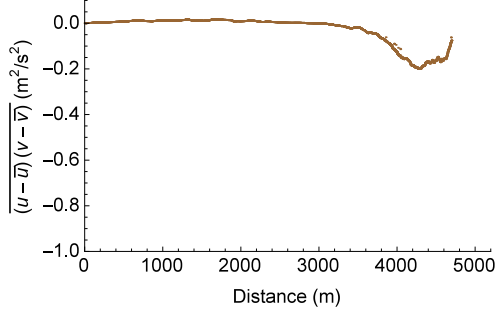

(a) $h_{\mathrm{m}}=0.5$

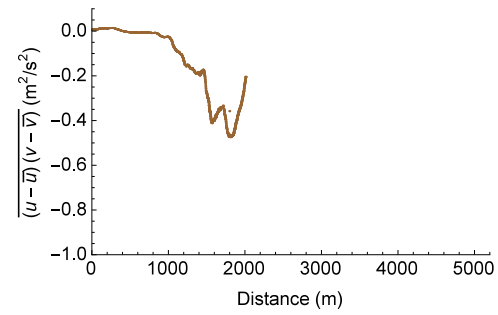

(d) CNS7 LF

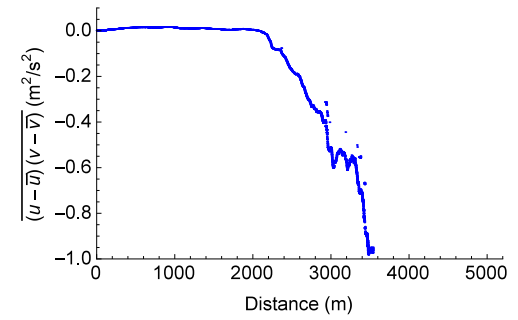

(g) CNS1 HF

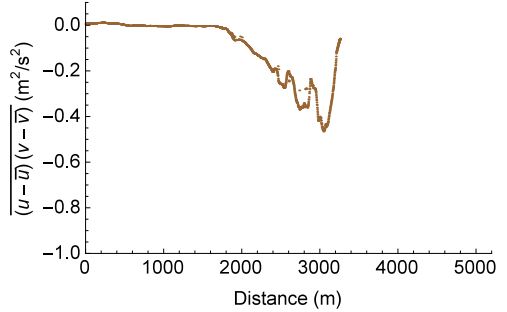

(b) CNS1 LF

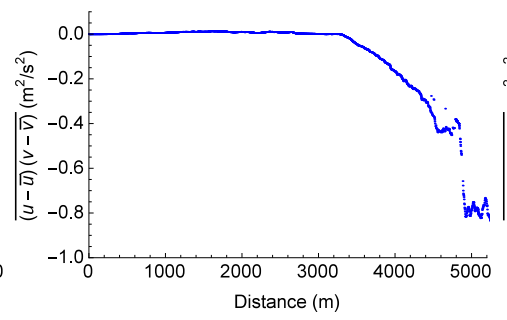

(e) $\mathrm{C} 8 \mathrm{HF}$

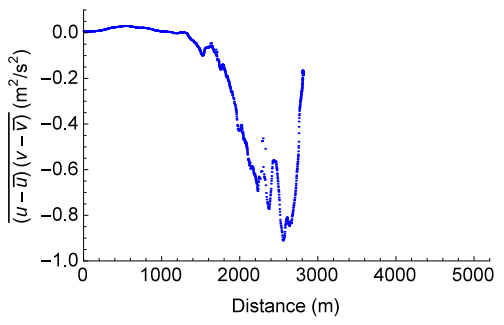

(h) CNS2 HF

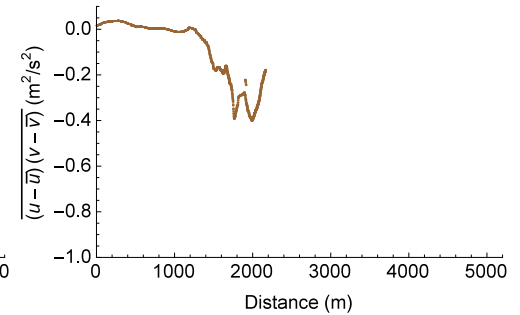

(c) CNS6 FL

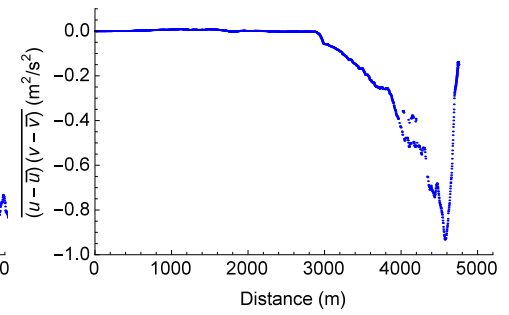

(f) $\mathrm{CNSO} \mathrm{HF}$

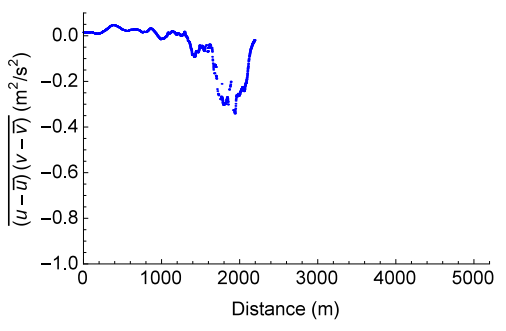

(i) CNS3 HF

Figure 7. Profiles of the momentum flux by the differential advection $\overline{(u-\bar{u})(v-\bar{v})}$ across the sampling transects. The brown and blue plots correspond to relatively low and high flow conditions, respectively.

\section{Conclusions}

This study examined the contributions of the depth- and length-averaged flow, turbulence, and differential advection to the lateral momentum flux at the confluence hydrodynamic zone (CHZ) of the Negro and Solimões rivers. The analyses were based on dense cross-sectional collections of secondary field data. The results confirm that the sharp bend of the Solimões, the flow convergence, the bed discordance between the tributaries, and the large Reynolds stress in the Solimões play active hydrodynamic roles in the significant moderation of the effect of the unstable shear layer on the momentum transfer and turbulent mixing. As a result, the lateral expansion of the large eddies located at the shear layers is limited. This partially explains the long distance required to complete the mixing of the waters of the two tributary rivers.

The momentum flux by the depth- and length-averaged flow is the dominant process of lateral momentum transfer; it is typically two orders of magnitude greater than the momentum flux by turbulence and one order of magnitude greater than the momentum flux by differential advection. These findings are similar to those of Rhoads et al. (2008) [5] obtained for a confluence with a width on the order of $10^{0}$ $\mathrm{m}$, whereas the width of the channels in the Negro-Solimões CHZ are on the order of $10^{3} \mathrm{~m}$. Lateral momentum fluxes in river confluences have a strong influence on the mixing of the flow itself, as well as the substances transported by the flow, over distances far beyond the $\mathrm{CHZ}$ (more than $100 \mathrm{~km}$ in 
the case of the Negro-Solimões confluence). Quantifying these fluxes has significance for engineering interventions, control of sediment and pollutants, evaluation of geomorphological patterns, and evaluation of aquatic habitat.

In confluences in straight rectangular channels, the lateral momentum transfer and the turbulence intensity are at the maximum in the center of the mixing interface, which is remarkably distinct from discordant converging confluences [15]. The maximum lateral momentum flux takes place in the far field of the Solimões side of the Amazon channel, associated with the sharp bend verified on this side of the channel. The magnitude of the momentum flux by turbulence is found to be well distributed across most of the Solimões side of the Amazon channel and not notably larger in the center of the mixing interface. This confirms that various large turbulent structures on the Solimões side, which is associated with complex channel geometry, coexist with the large eddies formed at the shear layer. Also, the effect of the shear layer on the transverse mixing is restricted to the center of the mixing interface because other turbulent structures inhibit its lateral expansion.

Acknowledgments: The author thanks Carlo Gualtieri for making data from the CLIM-Amazon Project available to the author in a convenient format.

Conflicts of Interest: The author declares no conflict of interest.

\section{References}

1. Brazilian Navy. Nautical Chart 4106B. 2013. Available online: https://www.marinha.mil.br/chm/dados-dosegnav / cartas-raster (accessed on 15 March 2015).

2. Laraque, A.; Guyot, J.L.; Filizola, N. Mixing processes in the Amazon River at the confluences of the Negro and Solimões Rivers, Encontro das Águas, Manaus, Brazil. Hydrol. Process. 2009, 23, 3131-3140. [CrossRef]

3. Brazilian National Water Agency (ANA). Hydrological Information System (Hidroweb). 2018. Available online: http://www.snirh.gov.br/hidroweb (accessed on 10 May 2018).

4. Roy, A.G.; Biron, P.M.; Buffin-Belanger, T.; Levasseur, M. Combined visual and quantitative techniques in the study of natural turbulent flows. Water Resour. Res. 1999, 35, 871-877. [CrossRef]

5. Rhoads, B.L.; Sukhodolov, A.N. Lateral momentum flux and the spatial evolution of flow within a confluence mixing interface. Water Resour. Res. 2008, 44, W08440. [CrossRef]

6. Tamai, N.; Asaeda, T.; Ikeda, H. Study on generation of periodical large surface eddies in a composite channel flow. Water Resour. Res. 1986, 22, 1129-1138. [CrossRef]

7. $\mathrm{Chu}, \mathrm{V} . \mathrm{H} . ; \mathrm{Wu}, \mathrm{J} .-\mathrm{H} . ;$ Khayat, R.E. Stability of transverse shear flows in shallow open channels. J. Hydraul. Eng. 1991, 117, 1370-1388. [CrossRef]

8. Ikeda, S.; Ohta, K.; Hasegawa, H. Instability-induced horizontal vortices in shallow open-channel flows with an inflection point in skewed velocity profile. J. Hydrosci. Hydraul. Eng. 1994, 12, 69-84.

9. Chen, D.; Jirka, G.H. Absolute and convective instabilities of plane turbulent wakes in a shallow water layer. J. Fluid Mech. 1997, 338, 157-172. [CrossRef]

10. Ghidaoui, M.S.; Kolyshkin, A.A. Linear stability analysis of lateral motions in compound open channel. J. Hydraul. Eng. 1999, 125, 871-880. [CrossRef]

11. Prooijen, B.C.; Uijttewaal, W.S.J. A linear approach for the evolution of coherent structures in shallow mixing layers. Phys. Fluids 2002, 14, 4105-4114. [CrossRef]

12. Kolyshkin, A.A.; Ghidaoui, M.S. Gravitational and shear instabilities in compound and composite channels. J. Hydraul. Eng. 2002, 128, 1076-1086. [CrossRef]

13. White, B.L.; Nepf, H.M. Shear instability and coherent structures in shallow flow adjacent to a porous layer. J. Fluid Mech. 2007, 593, 1-32. [CrossRef]

14. Lima, A.C.; Izumi, N. Spatial and temporal growth of perturbations in open-channel viscous transverse shear flow. J. Hydraul. Eng. 2016, 142, 04016067. [CrossRef] 
15. Lima, A.C.; Izumi, N. On the nonlinear development of shear layers in partially vegetated channels. Phys. Fluids 2014, 26, 084109. [CrossRef]

16. Gualtieri, C.; Filizola, N.; Oliveira, M.; Santos, A.M.; Ianniruberto, M. A field study of the confluence between Negro and Solimões Rivers. Part 1: Hydrodynamics and sediment transport. Comptes Rendus Geosci. 2018, 350, 31-42. [CrossRef]

17. Ianniruberto, M.; Dantas, E.; Pinheiro, A.; Trevethan, M.; Filizola, N.; Santos, A.; Fernando, J.; Gualtieri, C. A field study of the confluence between Negro and Solimões Rivers. Part 2: Riverbed morphology and stratigraphy. Comptes Rendus Geosci. 2018, 350, 43-54. [CrossRef]

18. Vreugdenhil, C.B. Numerical Methods for Shallow-Water Flow; Kluwer Acad.: Dordrecht, The Netherlands, 1994.

19. Mosley, P. An Experimental study of channel confluences. J. Geol. 1976, 84, 535-562. [CrossRef]

20. Best, J. Flow dynamics at river channel confluences: Implications for sediment transport and bed morphology. In Recent Developments in Fluvial Sedimentology; Ethridge, F., Flores, M., Harvey, M., Eds.; Society of Economic Paleontologists and Mineralogists: Tulsa, OK, USA, 1987; pp. 27-35.

21. Trevethan, M.; Martinelli, A.; Oliveira, M.; Ianniruberto, M.; Gualtieri, C. Fluid mechanics, sediment transport and mixing about the confluence of Negro and Solimões rivers, Manaus, Brazil. In Proceedings of the 36th IAHR World Congress, The Hague, The Netherlands, 28 June-3 July 2015.

22. Nordin, C.F.; Meade, R.H.; Mahoney, H.A.; Delaney, B.M. Particle Size of Sediments Collected from the Bed of the Amazon River and Its Tributaries in June and July 1976; USGS: Denver, CO, USA, 1977.

23. Wilcock, P.R. Estimating local shear stress from velocity observations. Water Resour. Res. 1996, 3361-3366. [CrossRef] 